technology on a more respectable footing in Britain as well as supplementing the work of economic and social historians. Much depends on the degree of interest which can be aroused among scientists and technologists, without whose assistance it will be virtually impossible to go on to specialized surveys of particular industries. The Committes already includes archrologists, historians, architects, geologists and technologists, but would warmly welcome the co-operation of more people with specialized knowledge of mining, quarrying, textiles, metallurgy, chemicals, civil and mechanical engineering or any of the other innumerable subjects in this vast field of industrial archæology.
E. R. R. GREeN

\title{
NINTH CANADIAN HIGH POLYMER FORUM
}

T HE ninth Canadian High Polymer Forum, held at the Guild Inn, Toronto, during October 26-28, was attended by 142 chemists from Great Britain, the United States and Canada. The chairman was M. H. Jones (Ontario Research Foundation) and the session chairmen were S. Bywater (National Research Council), H. H. G. Jellinek (Eissex College), M. Rinfret (University of Montreal) and G. Olah (Dow of Canada).

At the business meeting on October 26 the officers for the tenth Forum were elected: chairman, L. A. McLeod, Polymer Corporation; programme chairman, K. E. Russell, Queen's University, Kingston, Ontario ; secretary-treasurer, D. A. I. Goring, Pulp and Paper Research Institute.

At the banquet on October 27, the guest speaker, C. H. Bamford, of Courtaulds, Maidenhead, spoke on "Patterns in Reactions and Polymers". He said that detailed information is now available concerning transfer reactions of polymer radicals with a variety of substrates. For a hydrocarbon substrate such as ethylbenzene, the rate constants for the hydrogen abstraction process involving a series of polymer radicals are fairly accurately proportional to the rate constants for the corresponding reactions with toluene. In these reactions with hydrocarbon substrates, polar factors are very small. With ferric chloride, butyl mercaptan or carbon tetrabromide as substrates, a logarithmic plot of rate constants for the transfer reaction against rate constants for the corresponding reaction with toluene gives a number of scattered points. The pattern of points is, how ever, very much the same in all three cases. With triethylamine as substrate an 'inverse' pattern is obtained. Patterns of the first type are obtained in addition reactions involving the same polymer radicals and a number of vinyl monomers.

The observed patterns can be interpreted in terms of polar effects, the rate constant depending considerably on the extent to which the substituent in the radical is electron-withdrawing or eloctronreleasing. From a quantitative treatment, Dr. Bamford concluded that it is possible to express the rate constant for the transfer reaction of a radical with a given substrate in terms of the rate constant for transfer with toluene and the Hammett $\sigma$ constant for the radical. Predictions based on this quantitative theory are in excellent agreement with experiment.

The main sessions began with a paper by J. K. N. Jones (Queen's University) on "Some Problems in Polysaccharide Chemistry". In pointing out some of the difficulties involved in complete structural determinations of polysaccharides, he said that it is not always possible to decide whether traces of sugars occurring with the main hydrolysis products of polysaccharides were part of the original macromolecule.
Degrees of branching can be readily obtained using the Haworth methylation technique, but frequently it is almost impossible to achieve 100 per cent methylation of a polysaccharide. The major problem in this field is to imitate the action of enzymes on sugars and sugar derivatives to produce polymers similar to those found in plants and animals.

B. L. Funt and F. D. Williams (University of Manitoba) in their paper on "Retardation and Inhibition of Methyl Methacrylate Polymerization" described investigations of the effect of benzoquinone on methyl methacrylate polymerization using quinone and initiator labelled with earbon-14. They observed a transition from retardation to inhibition at a critical concentration of benzoquinone. The Monday morning session closed with a short colour film on polymer interactions made available by W. F. Busse, Du Pont Co., Wilmington.

The first in a series of papers on polymer degradation was given by H. A. Kraessig and J. Neal (Industrial Cellulose Research), who discussed "The Degradation of Cellulose by Megavolt Electrons". They showed that at low dose-rates the number of chemical bonds broken is a linear function of the dose, and the sensitive volume corresponding to cleavage of a single bond is close to the volume of a monomer unit. Experiments on wet cellulose indicate that water has a considerable protective action. H. H. G. Jellinek (Essex College) and W. A. Schlueter described work performed at the University of Cincinatti on "The Photodegradation of Polyacrylonitrile in Solution", using light of wave-length $2537 \AA$. The rate of degradation in vacuo is proportional to the light intensity and the number of broken bonds is less than $10^{-8}$ per quantum absorbed. W. C. Schneider (American Cyanamid) spoke on "The Thermal Stability of Polyacrylonitrile". At $240^{\circ}$ in the absence of oxygen he observed that the polymer breaks down very rapidly giving a complex mixture of products, but in the presence of oxygen a more controlled reaction gives water, ammonia and hydrogen cyanide as the only volatile products. J. R. Schaefgen (Du Pont) presented a paper on "The Thermal Degradation of Poly- $p$-xylylene". The rate of degradation was measured in a chlorinated aromatic solvent in the range $285-321^{\circ}$. Degradation is random, and the activation energy is $58 \mathrm{kcal}$./mole. Anthracene decreased the rate of degradation, but common transfer agents are inactive.

R. J. Orr (Polymer Corporation) presented a paper on "Thermochemical Aspects of Free Radical Butadiene-Styrene Copolymerization". A treatment of the thermodynamics of copolymerization has been devised which can be applied to high-conversion material. The sum of the entropy changes for the two heteropolymerization steps was found to be 
about 50 entropy units/mole assuming that the residual entropy contributed by the glassy state is zero.

C. Booth and L. R. Beason (Shell Chemical) gave a paper on "Statistical Treatment of Polymer Fractionation Data". A styrene-butadiene copolymer was fractionated and the fractionation data subjected. to a simple binomial analysis. The resulting distribution of molecular weight was in good agreement with that calculated from the kinetics of the emulsion system. J. A. Manson, W. W. Graessley and L. M. Hobbs (Air Reduction Co.) described "The Determination of the Breadth of Molecular Weight Distribution in Polyethylene by an Irradiation Technique". Samples of high-pressure and lowpressure polyethylene were irradiated using a cobalt60 source and were analysed for soluble and gel fractions. From the results it is possible to calculate weight and number average molecular weights of the original polyethylene samples.

A. Rezanowich and D. A. I. Goring (Pulp and Paper Research Institute) have investigated "The Polyelectrolyte Properties of a Lignin Sulphonate Microgel". Their measurements of the polyelectrolyte expansion of fractions of sodium lignin sulphonate indicate a microgel structure for the macromolecule. They developed a theory assuming that the microgel structure has free charges only on the surface and showed that it agreed fairly well with experiment.

D. J. Worsfold and S. Bywater (National Research Council) spoke on "Some Properties of Poly- $\alpha$ methylstyrene produced by Anionic Polymerization". They have made a thorough molecular weight analysis of poly- $\alpha$-methylstyrene samples produced using sodium naphthenide as catalyst. One important result is the intrinsic viscosity-molecular weight relationship for polymer samples of very sharp distribution.

M. Senez and H. Daoust (University of Montreal), in their paper on "Heat Parameters for Polyisobutylene Solutions", compared heat parameters determined for the polyisobutylene-chlorobenzene system by calorimetry and by the viscosity method of Fox and Flory. The results fit the Fox and Flory treatment of viscosity data.

W. Heller and M. Nakagaki (Wayne State University), in "The Exact Theory of the Scanning Method for determining Particle Sizes from Light Scattering", gave accurate calculations of the angular positions of maxima and minima for non-absorbing spheres for the large relative refractive index of 1.20. The results for other relative refractive indexes can now be calculated fairly readily.

T. Gillespie (Dow Chemical) presented papers on "The Limited Flocculation of a Colloidal System by a Water Soluble Polymer" and "The Rheology of a Polystyrene Latex thickened with Methylcellulose". His results on the flocculation of styrene-butadiene latex by methylcellulose indicate that the stabiliza. tion of a hydrophobic colloid by a hydrophilic colloid is due to changes in the balance of the flocculation and deflocculation processes. Methylcellulose thickens a polystyrene latex by causing a reversible partial flocculation.

A. Novak and E. Whalley (National Research Council) presented data on "The Infra-red Spectrum of Polyformaldehyde" and showed that these data could be interpreted on the assumption of a helical model for the polymer.

E. H. Immergut, G. Kollmann and A. Malatesta (Dunlop Research Centre) reported results on "The Cationic Copolymerization of Propylene and Iso- prene". They prepared soluble copolymers under carefully controlled conditions using aluminium chloride as catalyst at $-78^{\circ}$ (molecular weight range $4,000-10,000)$. Reactivity ratios for propylene and isoprene are approximately 0.23 and 0.5 . M. H. Jones, U. Martius and M. P. Thorne (Ontario Research Foundation) discussed "The Polymerization of 1-Butene by Metal Alkyl-Titanium Halide Catalysts". They reported the results of chemical and X-ray analyses of the complex catalysts produced by the interaction of aluminium triisobutyl or lithium $n$-butyl with titanium trichloride or tetrachloride. Kinetic results for the polymerization of butene-1 using aluminium triisobutyl-titanium trichloride were given; the products were of very high molecular weight (intrinsic viscosity between 5 and 6 ).

C. H. Bamford, in a paper on "Termination by Primary Radicals in Vinyl Polynerization", said that when the initiator concentration is high, a plot of rate of polymerization of styrene versus monomer concentration is curved. This can be quantitatively accounted for by assuming termination of some growing polymer radicals by primary radicals from the initiator. The velocity constant for primary radical termination is about sixty times that for mutual termination. The interaction of unlike radicals was also discussed by $\mathrm{C}$. Sivertz and $\mathrm{Y}$. Ebisuzaki (University of Western Ontario) in a paper on "The Measurement of the Cross-Termination Velocity Constants for Picrylhydrazyl and Alkyl Radicals". They studied the thermal decomposition of azobisisobutyronitrile at such low concentrations that the combination of free cyano-alkyl radicals competed with the reaction with diphenylpicrylhydrazyl. The relationship between the two velocity constants was derived.

L. E. Coleman and J. F. Bork (The Lubrizol Corporation) spoke on "The Reactivity Ratios of N-Vinyl Oxazolidone and N.Vinyl Pyrrolidone with Vinyl Monomers". N-Vinyl oxazolidone copolymerizes well with vinyl chloride and vinyl acetate but not with styrene and methyl methacrylate; $\mathrm{N}$-vinyl pyrrolidone is more reactive than $\mathrm{N}$-vinyl oxazolidone. W. E. Walles, W. F. Tousignant and T. Houtman (Dow Chemical) have studied "Poly-N-vinyl, 5-Methyl 2-Oxazolidone (PVOM) a New Complexing Polymer". PVOM of molecular weight 150,000 is soluble in water below $40^{\circ}$ and insoluble at higher temperatures. This behaviour is explained in terms of a change from a structure which presents largely hydrophilic groups to the solvent to one which is almost entirely hydrophobic.

R. J. Ceresa and F. H. Cotton (National College of Rubber Technology, London), in presenting a paper on "The Mechanico-Chemical Modification of High Polymers", said that cold mastication of elastomers in the presence of 0.1 per cent aluminium isopropoxide eventually leads to gels; under similar conditions a mixture of polyvinyl acetate and poly. ethylene may give a fair yield of block copolymer.

R. J. Richardson (Atomic Energy of Canada) spoke on "Radiation Grafting of High Polymers". Nylon tenting fabric was irradiated with gamma-rays in the presence of styrene to give polystyrene grafts largely on the surface of the nylon, with a resultant improvement in its weathering properties. Irradiation of nylon and terylene in the presence of acrylonitrile and vinyl pyrrolidone gave a slight improvement in dyeing response.

J. R. Tichy (Maine Medical Center), in his paper on "The Polymerization of Phosphorus Pentachloride 
and Urea", presented analytical data on the products of this complex polymerization. In the last paper presented, R. W. Lenz and W. K. Carrington (Dow Chemical) discussed "The Preparation of Phenylene Sulphide Polymers by the Macallum Polymerization". The reaction of $p$-dichlorbenzene with sulphur and sodium carbonate at $300-350^{\circ}$ to give a high molecular weight phenylene sulphide polymer probably proceeds partly by a direct attack of sulphur diradicals on the dihalide and partly by attack of sodium sulphide formed by reaction of sulphur with sodium carbonate.

K. E. Russert

\section{DIELECTRIC DEVICES}

$\mathrm{A}$ $\mathrm{N}$ informal residential conference on dielectric devices was held in the Electrical Engineering Department of the University of Birmingham during September 14-17, 1959, inclusive, and was attended by representatives from universities, Government establishments and industry. The aims of the conference were to give those participating a broad overall picture of this sector of solid-state physical electronics, to discuss present trends and new ideas connected with the investigation and exploitation of dielectrics, and to stimulate research on dielectric materials and devices. In an attempt to achieve these aims each session was opened with an invited general paper which reviewed physical principles and assessed practical progress in exploitation; each session then continued with shorter papers describing current research on the properties of materials and the mechanisms of devices.

The first paper of the conference opened the session on masers and parametric amplifiers. This was given by I. M. Ross (Services Electronics Research Laboratories, Harlow), who reviewed the mechanisms of these devices and compared their more important characteristics such as gain bandwidth products and noise figures. J. C. Walling (Mullard Research Laboratories, Salfords) then described the paramagnetic resonance characteristics of chromium-doped potassium cobalticyanide and the advantages of maser operation using 'push-pull' and 'push-push' pumping. Experimental realization of both types of double pumping had been obtained in a cavity maser at a signal frequency of $2,900 \mathrm{Mc}$. $\mathrm{s}$. with respective root-gain bandwidth products of 12 and $19 \mathrm{Mc} / \mathrm{s}$. in the two cases.

In the following paper, by B. Bölger, N. J. Robinson and J. Ubbink (Philips, Eindhoven), read by $J$. Ubbink, the characteristics of a $1,420 \mathrm{Mc} / \mathrm{s}$. maser were described. A three-level maser using potassium cobalticyanide at $1.4^{\circ} \mathrm{K}$, with a pump frequency of $3,850 \mathrm{Mc}$./s. and a signal frequency of $1,420 \mathrm{Mc} . / \mathrm{s}$. had been constructed. A root-gain bandwidth product of $2.7 \mathrm{Mc} / \mathrm{s}$. with a limiting power of $2 \times 10^{-7} \mathrm{~W}$. had been obtained. The final paper of this session dealt with parametric amplification and was given by L. E. Cross (Electrical Research Association, Leatherhead), who considered the use of a ferroelectric material above its Curie temperature as a possible variable reactance for this purpose. The electro-mechanical effects in ferro-electrics wore briefly discussed and saturation functions derived for barium titanate and glycine sulphate. Measurements showed that glycine sulphate could not be used at $500 \mathrm{Mc}$./s. duo to enhanced diolectric losses near the Curie temperature.

The second session was openod by G. F. J. Garlick (University of Hull), who reviewed the physical mechanisms of photoconductivity and luminescence. H. G. Lubszynski (E.M.I., Hayes) then explained how the use of an electron beam for making contact to a semi-conductor offers advantages in the case of near insulators because it allows thin films of large cross-sectional area to be used with less danger of shorting. Organic luminescence and the scintillation counter formed the subjects of the next two papers. In the first of these, J. B. Birks (University of Manchester) discussed the fast and slow components of scintillations in organic materials. The former was attributed to direct singlet-state excitation, was subject to primary quenching, and hence depended on the nature of the particle. The latter was attributed to ion recombination leading to excitation of metastable triplet states, was less subject to primary quenching, and was largely independent of the nature of the particle. P. E. Gibbons and D. C. Northrop (Services Electronics Research Laboratories, Baldock) then described experiments on the longlived decay components of anthracene scintillations showing that the characteristic times are independent of exciting particles and temperature. These and other experiments on impure anthracene show that these components probably derive from some cooperative lattice property and not a molecular one.

The opening paper of the third session was given by G. Diemer (Philips, Eindhoven), who reviewed present and potential applications of luminescence and photoconductivity in power amplification. The first research paper of the session was read by $P . K$. Weimer for E. E. Loebner (R.C.A., Princeton) and explained how it should be possible to escape the gain-bandwidth limitations of currently available luminescence amplifiers by using luminor elements themselves capable of modulation and amplification. The second research paper was given by J. Woods (G.E.C., Wembley), who described experiments showing that cadmium sulphide crystals doped with 1-2 per cent copper are $p$-type. Measurements of electrical conductivity, $\sigma$, Hall coefficient, $R$, and thermoelectric power, $\alpha$, had been made from $95^{\circ} \mathrm{K}$. to $380^{\circ} \mathrm{K}$., and suggested transport via impurity-levels. At $300^{\circ} \mathrm{K}$. it was found for $\sigma=0.18 \mathrm{ohm}^{-1} \mathrm{~cm} .^{-1}$ that $p=1 / R e=5.6 \times 10^{18} \mathrm{~cm}^{-3}, \mu=R \sigma=20 \mathrm{~cm}^{2}$ volt $^{-1}$ sec. ${ }^{-1}$, and $\alpha=22 \mu \mathrm{V} .1{ }^{\circ} \mathrm{C}$.

Space-charge-limited current in dielectric crystals formed the topic for the fourth session. The opening paper of this session was given by G. T. Wright (University of Birmingham) and reviewed the mechanisms of space-charge-limited current in dielectrics and, on the basis of a simplified theory and available experimental data, assessed tentatively potential fields of application of space-chargelimited dielectric devices. In a short following paper, A. A. Kayali (University of Birmingham) described measurements of the temperature dependence of forward current of a number of space-chargelimited cadmium sulphide dielectric diodes. In diodes with a small trap density the current increased slowly as the temperature was lowered from $400^{\circ} \mathrm{K}$. to $90^{\circ} \mathrm{K}$. due to the increase of electron mobility at 